\title{
The Oscillation of a System Earth - Moon
}

\section{G. Kurbasova}

Crimea Astrophysical Observatory, Ukrainian Academy of Sciences, $p / o$ Nauchnyi, Crimea, 334413 Ukraine, e-mail: gsk@crao.crimea.ua

\section{Rykhlova}

Institute for Astronomy, Russian Academy of Sciences, 109017

Pyatnitskaya Str.48, Moscow,Russia.e-mail:rykhlova@inasan.rssi.ru

\section{Summary}

Internal links in the Earth-Moon system are determined by gravitational interaction. According to the least compulsion principle of Gauss, the deviation of "free motion" of heliocentric orbits of two material points with Earth and Moon masses is determined by the sum of the products of each material points' deviation from its free motion and its mass.

By solving the minimization problem using the Lagrange multiplier method, Lagrange equations of the first kind were obtained in vector form. With acceptable assumptions (introduction of non-dimensional time $\tau=n t$, where $\mathrm{n}$ is the sidereal rotation of the Moon, and the lunar orbit is considered to be circular) the linkage coefficient (Lagrange multiplier) is:

$$
\lambda=-\left(\nu_{1}^{2}-\nu_{2}^{2}\right) \frac{1+\mu}{1-\mu}
$$

where $\nu_{1}$ and $\nu_{2}$ are proper frequencies of the Earth and the Moon and $\mu$ is the Moon/Earth mass ratio.

The linkage degree of the physical system (Mandelshtam, 1972) in this case is defined by the parameter

$$
\sigma=2 \frac{1+\mu}{1-\mu}
$$

To describe the relative motion we consider equations for small deviations from the Earth-Moon mass-center.

Assuming that between the Earth and the Moon there are no translational motions and that variables are the angular velocities of the bodies, a system of equations with two degrees of freedom is obtained. When choosing the idealized system, the features of the real motion were taken into account as well as the possibility of neglecting those frequencies of the real features that differ substantially from the frequencies of the considered oscillations. The flattening of the Earth is accounted for by replacing its mass by two mass-points situated at a distance of two Earth radii.

As a result of the solution of this system of equations the following relation between frequencies of the individual bodies and oscillations of the Earth-Moon system is: 


$$
\omega_{1,2}^{2}=\frac{\nu_{1}^{2}+\nu_{2}^{2}}{2} \mp \frac{\left|\nu_{1}^{2}-\nu_{2}^{2}\right|}{2} \sqrt{1+\sigma^{2}} .
$$

According to this relation of motions of individual bodies with periods $P_{1}=$ 403.3 days and $P_{2}=365.25$ days, corresponding oscillations in the Earth-Moon system have periods $T_{1}=435$ days and $T_{2}=346$ days.

\section{References}

Mandelshtam L. I, 1972, Complete set of the transaction, Vol IV, Moscow: Nauka, 512 pp.

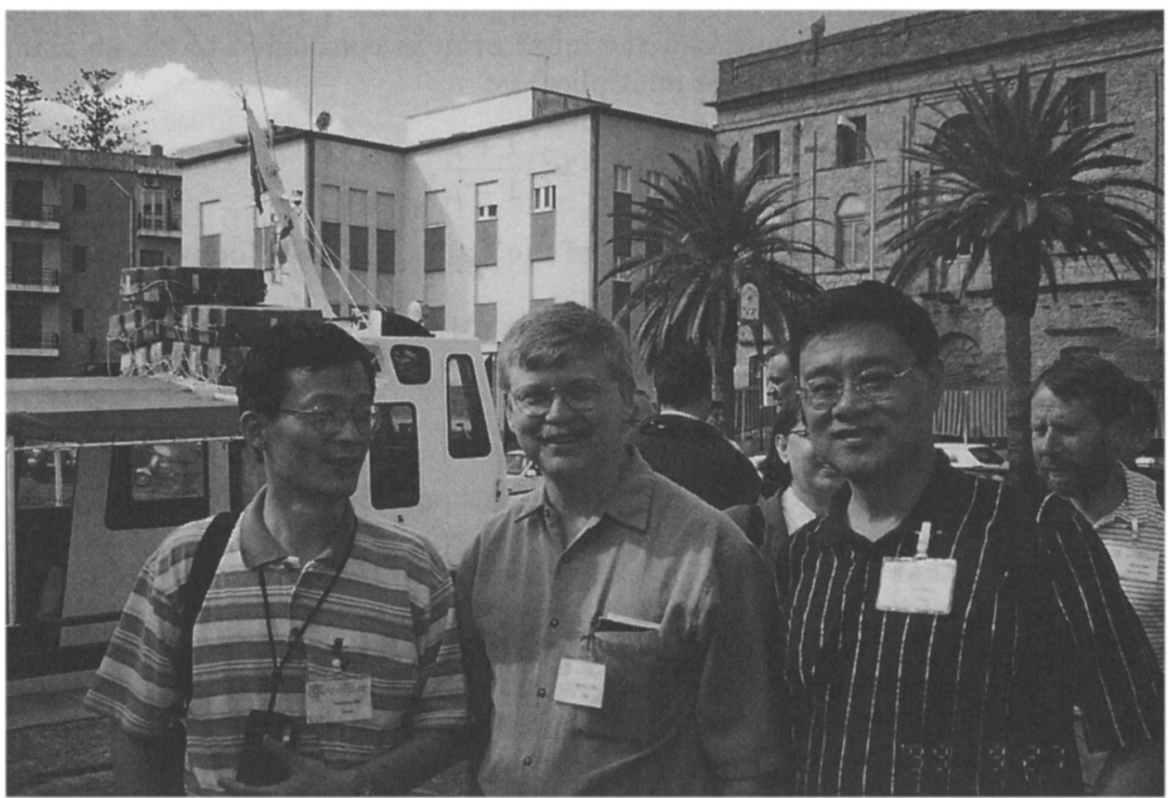

Y. Zhu, Steve Dick, and Wang Wen Jun 